\title{
Classification of Numerical 3-Semigroups by means of L-shapes*
}

\author{
Francesc Aguiló and Carlos Marijuán \\ (matfag@ma4.upc.edu, marijuan@mat.uva.es) \\ Dept. Matemàtica Aplicada IV, U. Politècnica de Catalunya, Barcelona, \\ Dpto. Matemática Aplicada, U. de Valladolid, Valladolid.
}

\begin{abstract}
We recall L-shapes, which are minimal distance diagrams, related to weighted 2-Cayley digraphs, and we give the number and the relation between minimal distance diagrams related to the same digraph. On the other hand, we consider some classes of numerical semigroups useful in the study of curve singularity. Then, we associate L-shapes to each numerical 3-semigroup and we describe some main invariants of numerical 3-semigroups in terms of the associated L-shapes. Finally, we give a characterization of the parameters of the L-shapes associated to a 3-numerical semigroup in terms of its generators, and we use it to classify the numerical 3-semigroups of interest in curve singularity.
\end{abstract}

Keywords: numerical semigroup, symmetric, free, curve plane semigroup, Cayley digraph, L-shape, Frobenius number.

\section{Introduction}

We are interested in numerical semigroups useful in the study of curve singularity, which receive the name of the corresponding classes of associated curves. Combinatorial objects, as simplicial complexes [4], have been introduced to study some geometrical properties of these numerical semigroups. But the particular numerical semigroups dealt with here will be introduced arithmetically and they will be considered as pure arithmetical objects.

The 2-Cayley digraphs have been widely used to study metrical applications of local area networks. Their generalization to weighted 2-Cayley digraphs, adding weights to the arcs, allows other applications to be studied. Looking for paths of minimum length in these digraphs, periodical plane tessellations with L-shaped tiles appear in the bibliography [7, 6]. Particular classes of L-shapes, so called minimal distance diagrams, have been used to study certain distance properties in 2-Cayley digraphs, and have also been associated to numerical 3 -semigroups $[10,2]$.

These particular L-shapes associated to a numerical 3-semigroup contain relevant information about the semigroup and, in fact, some main invariants of the semigroup, such as Apéry sets, Frobenius number, set of gaps ..., can be described in terms of the associated L-shapes.

${ }^{*}$ Work supported by the Comisión Interministerial de Ciencia y Tecnología MCYT ref. MTM2007-64704C03-01, MTM2012-36917-C03-01 and MTM2011-28800-C02-01 and the Catalan Research Council under grant 2009SGR1387. 
Our goal in this work is to show the close relation between numerical 3-semigroups, which are interesting in the mentioned geometric context, and their associated L-shapes, in such a way that we can describe problems in a numerical 3-semigroup in terms of the associated L-shapes, and vice-versa.

Our last objective is to understand in depth the relations between semigroups and L-shapes to try to understand some complex problems in geometry by means of these combinatoric tools, which are not so complex as those used in geometry [4]. In particular, we try to understand complete intersection semigroups [5, 9] which, for $n \geq 4$, are distinguished from symmetric semigroups. A thorough knowledge of the case $n=3$ will allow us to approach the case $n=4$.

In Section 2, we introduce L-shapes related to weighted 2-Cayley digraphs and minimum distance diagrams in this context. In section 3 , we study the number and the relation between the minimum distance diagrams related to the same digraph. In Section 4, we introduce numerical semigroups interesting in the study of curve singularity and we describe some invariants of numerical 3-semigroups in terms of their associated L-shapes. Finally, in Section 5 we characterize these numerical 3-semigroups in terms of the number and the type of their associated L-shapes.

\section{L-shaped minimum distance diagrams}

In this section we introduce L-shapes related to certain weighted 2-Cayley digraphs and the particular cases of minimum distance diagrams. These geometrical objectes will be used to obtain main information about their related semigroups.

For integers $g, n \in \mathbb{N}$, let us denote the equivalence class of $g$ modulo $n$ by $[g]_{n}$. Given integers $a, b, c \in \mathbb{N}, 1 \leq a<b<c, \operatorname{gcd}(a, b, c)=1$ and weights $W_{a}, W_{b} \in \mathbb{R}^{+}$, a weighted 2-Cayley digraph $G\left(c ; a, b ; W_{a}, W_{b}\right)=\operatorname{Cay}\left(\mathbb{Z}_{c} ; a, b ; W_{a}, W_{b}\right)$ is a directed graph with sets of vertices $V(G)=\mathbb{Z}_{c}$ and $\operatorname{arcs} A(G)=\left\{[g]_{c} \stackrel{W_{a}}{\longrightarrow}[g+a]_{c},[g]_{c} \stackrel{W_{b}}{\longrightarrow}[g+b]_{c}: g=0, \ldots, c-1\right\}$, where $\stackrel{W_{a}}{\longrightarrow}$ and $\stackrel{W_{b}}{\longrightarrow}$ stands for weighted arcs with related weights $W_{a}$ and $W_{b}$, respectively. In this context we consider $\mathbb{Z}_{c}$ generated by the set $\{a, b\}$. We denote the distance from vertex $[g]_{c}$ to vertex $[h]_{c}$ in $G$ by $\mathrm{d}_{G}\left([g]_{c},[h]_{c}\right)$ (the weight of a minimal weighted path from $[g]_{c}$ to $\left.[h]_{c}\right)$. From now on, we simplify "weighted 2-Cayley digraph $G\left(c ; a, b ; W_{a}, W_{b}\right)$ " to "digraph $G\left(c ; a, b ; W_{a}, W_{b}\right)$ ". Metrical properties of these digraphs have been widely studied using "minimum distance diagrams" $[7,6,11]$.

Let us consider unit squares $\llbracket i, j \rrbracket=[i, j] \times[i+1, j+1] \subset \mathbb{R}^{2}$ with integer non negative coordinates $(i, j) \in \mathbb{N}^{2}$. Each square $\llbracket i, j \rrbracket$ is related to the equivalence class $[i a+j b]_{c}$, and we denote the weight of the unit square $\llbracket i, j \rrbracket$ by $\delta(i, j)=i W_{a}+j W_{b}$. Given any integer $0 \leq n<c$, let us consider the set of unit squares in the first quadrant related to $[n]_{c}$

$$
Q_{n}=\left\{\llbracket i, j \rrbracket:[i a+i b]_{c}=[n]_{c}, 0 \leq i, j\right\},
$$

and the weight of $[n]_{c}$

$$
M_{n}=\min \left\{\delta(i, j): \llbracket i, j \rrbracket \in Q_{n}\right\}=\mathrm{d}_{G}\left([0]_{c},[n]_{c}\right) .
$$

An L-shape related to the digraph $G\left(c ; a, b ; W_{a}, W_{b}\right)$ consists of $c$ unit squares related to the equivalence classes $[n]_{c}, 0 \leq n<c$. L-shapes are denoted by the lengths of their sides, 
$L(l, h, w, y)$, with $0 \leq w<l$ and $0 \leq y<h$ as in the left hand side of Figure 1. Rectangles are considered degenerated L-shapes, i.e., L-shapes with $w y=0$. Conditions

$$
l h-w y=c \quad \text { and } \operatorname{gcd}(l, h, w, y)=1
$$

are necessary for an L-shape $L(l, h, w, y)$ to be related to a given digraph $G\left(c ; a, b ; W_{a}, W_{b}\right)$. It is also well known that an L-shape $L(l, h, w, y)$ periodically tessellates the plane by translation through the vectors $\boldsymbol{u}=(l,-y)$ and $\boldsymbol{v}=(-w, h)$ (right hand side of Figure 1). In terms of equivalence classes, this fact results in $[i a+j b]_{c}=[(i+l) a+(j-y) b]_{c}$ and $[i a+j b]_{c}=$ $[(i-w) a+(j+h) b]_{c}$, that is

$$
l a \equiv y b(\bmod c) \quad \text { and } \quad h b \equiv w a(\bmod c) .
$$
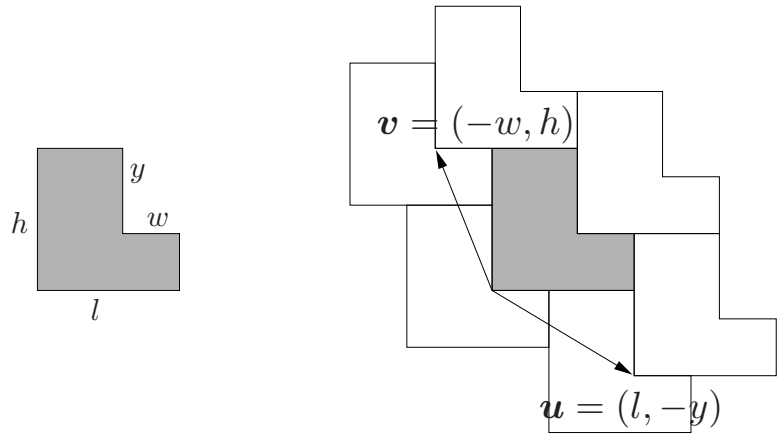

Figure 1: L-shape $L(l, h, w, y)$ and the related plane tessellation

Fiol et al. [7] showed that an L-shape $L(l, h, w, y)$ is related to the digraph $G\left(c ; a, b ; W_{a}, W_{b}\right)$ if and only if (1) and (2) hold.

Let $\Delta(i, j)$ be the set of unit squares in the first quadrant dominated by $\llbracket i, j \rrbracket$, i.e.

$$
\Delta(i, j)=\{\llbracket u, v \rrbracket: 0 \leq u \leq i, 0 \leq v \leq j\} .
$$

Definition 1 An L-shape related to $G\left(c ; a, b ; W_{a}, W_{b}\right)$ is a minimum distance diagram (MDD for short) if it satisfies the following two properties:

(a) For each $\llbracket s, t \rrbracket \in L$, identity $\delta(s, t)=M_{s a+t b}$ holds.

(b) If $\llbracket s, t \rrbracket \in L$ then $\Delta(s, t) \subset L$.

The following result is a geometrical characterization of minimum distance diagrams.

Theorem 1 ([3]) Let $\mathcal{H}=L(l, h, w, y)$ be an $L$-shape related to the digraph $G=G\left(c ; a, b ; W_{a}, W_{b}\right)$. Then $\mathcal{H}$ is an $M D D$ related to $G$ if and only if $l W_{a} \geq y W_{b}, h W_{b} \geq w W_{a}$ and both equalities do not hold at the same time.

Next section deals with properties of MDD related to general digraphs $G\left(c ; a, b ; W_{a}, W_{b}\right)$.

\section{Properties of MDDs related to a weighed 2-Cayley digraph}

In this section we study geometrical properties of minimum distance diagrams related to the same weighed 2-Cayley digraph. In particular, we give their cardinal and geometrical links 
between them.

From now on, given an L-shape $\mathcal{H}=L(l, h, w, y)$, we assume the left lower square of $\mathcal{H}$ is $\llbracket 0,0 \rrbracket$ by default. Set $\nabla(i, j)=\{\llbracket s, t \rrbracket: s \geq i, t \geq j\}, A_{\mathcal{H}}=\nabla(l-w, h-y), B_{\mathcal{H}}=\nabla(l, 0) \backslash \nabla(l, h-y)$ and $C_{\mathcal{H}}=\nabla(0, h) \backslash \nabla(l-w, h)$. Note the disjoint union $\mathbb{N}^{2}=\mathcal{H} \cup A_{\mathcal{H}} \cup B_{\mathcal{H}} \cup C_{\mathcal{H}}$ as it is depicted in Figure 2.

We use the notation $\llbracket i, j \rrbracket \sim \llbracket m, n \rrbracket$ whenever $[i a+j b]_{c}=[m a+n b]_{c}$.

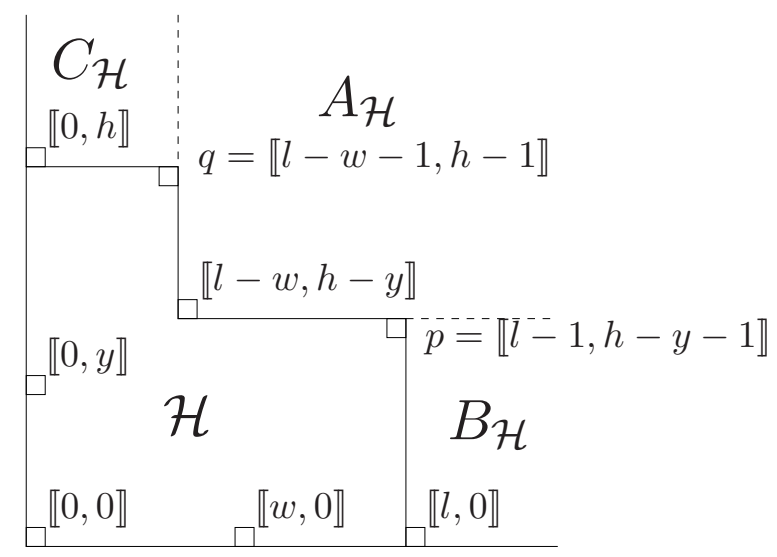

Figure 2: Regions $A_{\mathcal{H}}, B_{\mathcal{H}}, C_{\mathcal{H}}$ and some highlighted unit squares

Lemma 1 Let $\mathcal{H}=L(l, h, w, y)$ be an $M D D$ related to a digraph $G=G\left(c ; a, b ; W_{a}, W_{b}\right)$. Then,

(a) if $\llbracket i, j \rrbracket \sim \llbracket s, t \rrbracket$ with $\llbracket i, j \rrbracket \in \mathcal{H}$ and $\delta(i, j)<\delta(s, t)$, it follows that $\delta(m, n)>\mathrm{d}_{G}\left([0]_{c},[m a+\right.$ $n b]_{c}$ ) for all unit squares $\llbracket m, n \rrbracket \in \nabla(s, t)$,

(b) any other $M D D$ related to $G$ does not intersect region $A_{\mathcal{H}}$,

(c) if $l W_{a}>y W_{b}$, any other $M D D$ related to $G$ does not intersect region $B_{\mathcal{H}}$,

(d) if $h W_{b}>w W_{a}$, any other $M D D$ related to $G$ does not intersect region $C_{\mathcal{H}}$,

(e) if $l W_{a}=y W_{b}$, then $h W_{b}>w W_{a}$,

(f) if $h W_{b}=w W_{a}$, then $l W_{a}>y W_{b}$.

Proof: (a) is a consequence of the fact that any subpath of a minimum path in $G$ is also a minimum path.

(b) comes from the equivalence $\llbracket 0,0 \rrbracket \sim \llbracket l-w, h-y \rrbracket$, i.e. by (2). Thus, applying (a), any unit square $\llbracket s, t \rrbracket \in A_{\mathcal{H}}$ does not satisfy minimality condition $\delta(i, j)=M_{s a+t b}$ and so, it can not belong to any MDD related to $G$.

Consider the unit squares $\llbracket 0, y \rrbracket \in \mathcal{H}$ and $\llbracket l, 0 \rrbracket \notin \mathcal{H}$. From the equivalence $\llbracket 0, y \rrbracket \sim \llbracket l, 0 \rrbracket$ (by (2)), we can apply again item (a) to prove (c). The same argument to prove (d) by taking unit squares $\llbracket w, 0 \rrbracket \in \mathcal{H}$ and $\llbracket 0, h \rrbracket \notin \mathcal{H}$.

For (e) and (f), we have $h W_{b}>y W_{b}=l W_{a}>w W_{a}$ and $l W_{a}>w W_{a}=h W_{b}>y W_{b}$.

Note that if $w=0$, then $h W_{b} \neq w W_{a}$, and if $y=0$, then $l W_{a} \neq y W_{b}$. 
Following Theorem 1 and items (e) and (f) of Lemma 1, given any MDD related to some digraph $G\left(c ; a, b ; W_{a}, W_{b}\right)$, we discuss the three possible cases: either $l W_{a}=y W_{b}$ or $h W_{b}=$ $w W_{a}$ or $\left(l W_{a}-y W_{b}\right)\left(h W_{b}-w W_{a}\right)>0$.

Theorem 2 Let $\mathcal{H}=L(l, h, w, y)$ be an $M D D$ related to a digraph $G=G\left(c ; a, b ; W_{a}, W_{b}\right)$. If $\left(l W_{a}-y W_{b}\right)\left(h W_{b}-w W_{a}\right)>0$, then there is no other $M D D$ related to $G$.

Proof: From Lemma 1-(b), another MDD related to $G, \mathcal{H}^{\prime}$, only intersects region $B_{\mathcal{H}} \cup C_{\mathcal{H}} \cup$ $\mathcal{H}^{\prime}$. From Lemma $1-(\mathrm{c})$ and $(\mathrm{d})$, the MDD $\mathcal{H}^{\prime}$ only intersects $\mathcal{H}$. As $\mathcal{H}$ and $\mathcal{H}^{\prime}$ have the same area, it follows that $\mathcal{H}=\mathcal{H}^{\prime}$.

Theorem 3 Let $\mathcal{H}=L(l, h, w, y)$ be an $M D D$ related to a digraph $G=G\left(c ; a, b ; W_{a}, W_{b}\right)$. If $h W_{b}=w W_{a}$, then there is another $M D D \mathcal{H}^{\prime}$ related to $G$ given by

$$
\mathcal{H}^{\prime}= \begin{cases}L(w, 2 h-y, 2 w-l, h) & l<2 w, \\ L(w,(\lfloor l / w\rfloor+1) h-y, w-r, h) & l>2 w>0, l=\lfloor l / w\rfloor w+r, 0<r<w, \\ L(w, l h / w-y, 0, h) & l \geq 2 w>0, l=\lfloor l / w\rfloor w .\end{cases}
$$

Proof: Equivalence between unit squares defined by the compatibility equations (2), leads to the idea of equivalence between regions of the plane (those regions containing clusters of equivalent unit squares). This idea allows us to think of the L-shaped MDD $\mathcal{H}^{\prime}$ as a recomposition of pieces of $\mathcal{H}$, like a puzzle.

If $h W_{b}=w W_{a}$, from Lemma 1, another MDD related to $G, \mathcal{H}^{\prime}=L\left(l^{\prime}, h^{\prime}, w^{\prime}, y^{\prime}\right) \neq \mathcal{H}$, intersects the region $C_{\mathcal{H}}$, that is $\mathcal{H}^{\prime} \not \subset \mathcal{H}$ and $\mathcal{H}^{\prime} \subset \mathcal{H} \cup C_{\mathcal{H}}$.
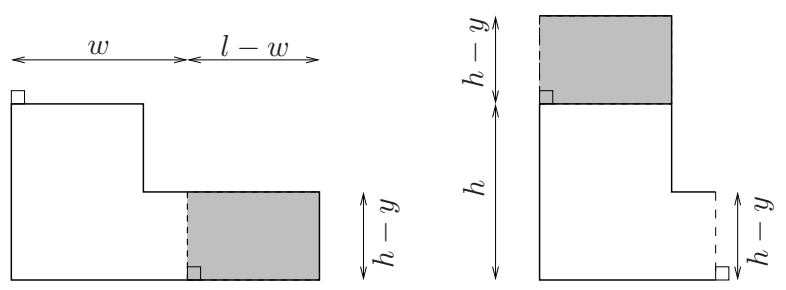

Figure 3: Case $l<2 w$. Diagrams $\mathcal{H}$ and $\mathcal{H}^{\prime}$, respectively

Note that $w \neq 0$. We consider the two cases, either $l<2 w$ or $l \geq 2 w>0$.

Case $\boldsymbol{l}<\mathbf{2 w}$ : Figure 3 shows which piece of $\mathcal{H}$ has to be considered to obtain $\mathcal{H}^{\prime}$. The two shaded pieces are equivalent regions, in the sense mentioned above, and they follow the distribution given by the vectors $\boldsymbol{u}=(l,-y)$ and $\boldsymbol{v}=(-w, h)(2)$. It follows that $l^{\prime}=w$, $h^{\prime}=2 h-y, w^{\prime}=2 w-l$ and $y^{\prime}=h$. So $\mathcal{H}^{\prime}=L(w, 2 h-y, 2 w-l, h)$.

We check now that $\mathcal{H}^{\prime}$ fulfills compatibility conditions (1) and (2), and Theorem 1 . Condition (1): clearly $l^{\prime} h^{\prime}-w^{\prime} y^{\prime}=c$, and $\operatorname{gcd}\left(l^{\prime}, h^{\prime}, w^{\prime}, y^{\prime}\right)=\operatorname{gcd}(w,-y, 2 w-l, h)=\operatorname{mcd}(w, y, l, h)=1$.

Condition (2) is also clear since $l^{\prime} a \equiv_{c} y^{\prime} b \Leftrightarrow w a \equiv_{c} h b$ and $w^{\prime} a \equiv_{c} h^{\prime} b \Leftrightarrow(2 w-l) a \equiv_{c}$ $(2 h-y) b \Leftrightarrow l a \equiv_{c} y b$.

Theorem 1: the first inequality $l^{\prime} W_{a} \geq y^{\prime} W_{b} \Leftrightarrow w W_{a} \geq h W_{b}$ is true because $h W_{b}=w W_{a}$; the second one because $h^{\prime} W_{b} \geq w^{\prime} W_{a} \Leftrightarrow(2 h-y) W_{b} \geq(2 w-l) W_{a} \Leftrightarrow l W_{a} \geq y W_{b}$.

Case $\boldsymbol{l} \geq \mathbf{2} \boldsymbol{w}>\mathbf{0}$ : Set $l=|l / w| w+r$, with $0 \leq r<w$. 

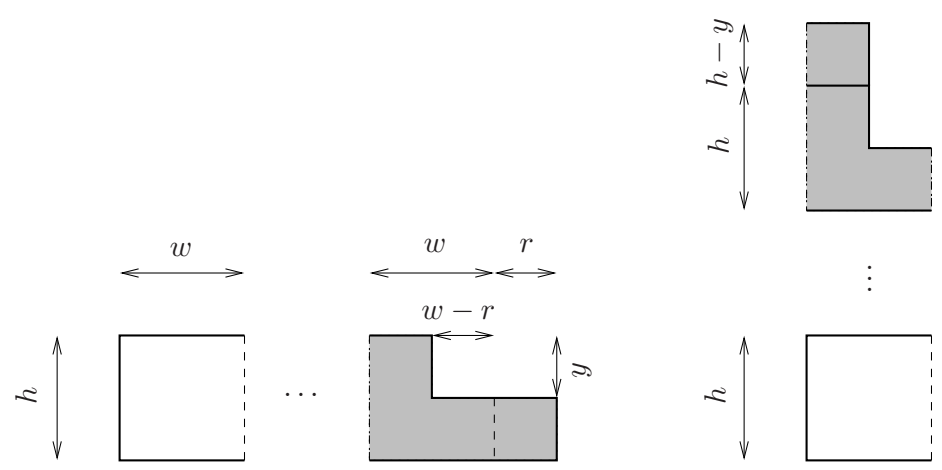

Figure 4: Case $l>2 w>0$ and $0<r<w . \mathcal{H}$ is divided in $\lfloor l / w\rfloor+1$ pieces

In the case $0<r<w$, see Figure 4, it follows that $l^{\prime}=w, h^{\prime}=\lfloor l / w\rfloor h+(h-y), w^{\prime}=w-r$ and $y^{\prime}=h$. So $\mathcal{H}^{\prime}=L(w,(\lfloor l / w\rfloor+1) h-y, w-r, h)$.

Condition (1):

$$
\begin{aligned}
l^{\prime} h^{\prime}-w^{\prime} y^{\prime} & =w[(\lfloor l / w\rfloor+1) h-y]-(w-r) h=(w\lfloor l / w\rfloor+r) h-w y=l h-w y=c, \\
\operatorname{gcd}\left(l^{\prime}, h^{\prime}, w^{\prime}, y^{\prime}\right) & =\operatorname{gcd}(w,(\lfloor l / w\rfloor+1) h-y, w-r, h)=\operatorname{gcd}(w,-y,-l, h)=1 .
\end{aligned}
$$

Condition (2): $l^{\prime} a \equiv_{c} y^{\prime} b \Leftrightarrow w a \equiv_{c} h b$, and

$$
\begin{aligned}
w^{\prime} a \equiv_{c} h^{\prime} b & \Leftrightarrow(w-r) a \equiv_{c}[(\lfloor l / w\rfloor+1) h-y] b \Leftrightarrow y b \equiv_{c}\lfloor l / w\rfloor h b+r a, \text { since } w a \equiv_{c} h b \\
& \Leftrightarrow w a \equiv_{c} h b, \text { since } y b \equiv_{c} l a=(\lfloor l / w\rfloor w+r) a
\end{aligned}
$$

Theorem 1: Inequality $l^{\prime} W_{a} \geq y^{\prime} W_{b} \Leftrightarrow w W_{a} \geq h W_{b}$ as in the case $l<2 w$. The second one because $\left.h^{\prime} W_{b} \geq w^{\prime} W_{a} \Leftrightarrow[(\lfloor l / w\rfloor+1) h-y] W_{b} \geq(w-r) W_{a} \Leftrightarrow h W_{b}=w W_{a}\right)(\lfloor l / w\rfloor h-$ y) $W_{b}+r W_{a} \geq 0$, which is true because $\lfloor l / w\rfloor \geq 2$.
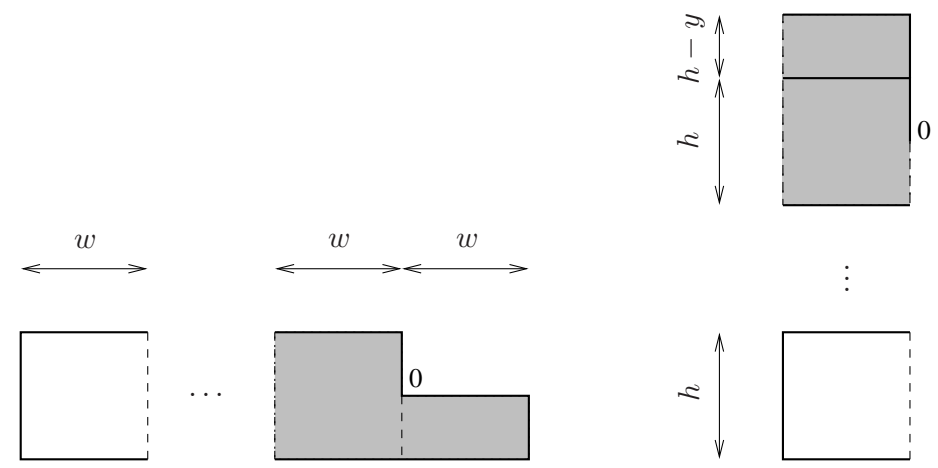

Figure 5: Case $l \geq 2 w>0$ and $r=0 . \mathcal{H}$ is divided into $l / w$ pieces

In the case $r=0$, see Figure 5, we have $l^{\prime}=w, h^{\prime}=(l / w-1) h+(h-y)=l h / w-y, w^{\prime}=0$ and $y^{\prime}=h$. So, $\mathcal{H}^{\prime}=L(w, l h / w-y, 0, h)$ (note the position of 0 in the figure).

Conditions (1): $l^{\prime} h^{\prime}-w^{\prime} y^{\prime}=w(l h / w-y)-0=l h-w y=c$, and

$$
\operatorname{gcd}\left(l^{\prime}, h^{\prime}, w^{\prime}, y^{\prime}\right)=\operatorname{gcd}(w, l h / w-y, 0, h)=\operatorname{gcd}(w,-y, h)=\operatorname{gcd}(w,-y, h, l)=1,
$$


Conditions (2): $l^{\prime} a \equiv_{c} y^{\prime} b \Leftrightarrow w a \equiv_{c} h b$, and

$$
w^{\prime} a \equiv_{c} h^{\prime} b \quad \Leftrightarrow \quad 0 \equiv_{c}(l h / w-y) b \Leftrightarrow y b \equiv_{c}(l / w) h b=l a,
$$

follow from $h b \equiv_{c} w a$ and $l a \equiv_{c} y b$.

Theorem 1: The first inequality, $l^{\prime} W_{a} \geq y^{\prime} W_{b}$, is fulfilled by the same reasons as in the previous case; the second one, $h^{\prime} W_{b} \geq w^{\prime} W_{a} \Leftrightarrow(l h / w-y) W_{b} \geq 0$, since $l / w \geq 2$.

Theorem 4 Let $\mathcal{H}=L(l, h, w, y)$ be an $M D D$ related to a digraph $G=G\left(c ; a, b ; W_{a}, W_{b}\right)$. If $l W_{a}=y W_{b}$, then there is another $M D D \mathcal{H}^{\prime}$ related to $G$ given by

$$
\mathcal{H}^{\prime}= \begin{cases}L(2 l-w, y, l, 2 y-h) & h<2 y, \\ L((\lfloor h / y\rfloor+1) l-w, y, l, y-r) & h>2 y>0, h=\lfloor h / y\rfloor y+r, 0<r<y, \\ L(l h / y-w, y, l, 0) & h \geq 2 y>0, h=\lfloor h / y\rfloor y .\end{cases}
$$

Proof: We can proceed analogously to the proof of Theorem 3. The MDD $\mathcal{H}^{\prime}$ is now formed by unit squares from $\mathcal{H}$ and the region $B_{\mathcal{H}}$. Note that if $l W_{a}=y W_{b}$, then $y \neq 0$.

From now on, we denote the transformations given in Theorem 3 and Theorem 4 by $\mathcal{T}_{1}(\mathcal{H})=$ $\mathcal{H}^{\prime}$ and $\mathcal{T}_{2}(\mathcal{H})=\mathcal{H}^{\prime}$, respectively.

Theorem 2 shows that there is a unique $\operatorname{MDD} \mathcal{H}=L(l, h, w, y)$ related to a digraph $G=$ $G\left(N ; a, b ; W_{a}, W_{b}\right)$ whenever $\left(l W_{a}-y W_{b}\right)\left(h W_{b}-w W_{a}\right)>0$. On the contrary, theorems 3 and 4 show that if $\left(l W_{a}-y W_{b}\right)\left(h W_{b}-w W_{a}\right)=0$, then $G$ has related more than one MDD. Moreover, in the latter case, we also see that each MDD can be obtained from the other by applying a suitable transformation, $\mathcal{T}_{1}$ or $\mathcal{T}_{2}$, given by either Theorem 3 or Theorem 4 . The last results in this section show that no more than two MDD are related to the same weighed 2-Cayley digraph.

Lemma 2 Let $\mathcal{H}=L(l, h, w, y)$ be an $M D D$ related to a digraph $G=G\left(c ; a, b ; W_{a}, W_{b}\right)$ with $\left(l W_{a}-y W_{b}\right)\left(h W_{b}-w W_{a}\right)=0$. If $\mathcal{H}^{\prime}=L\left(l^{\prime}, h^{\prime}, w^{\prime}, y^{\prime}\right)$ is the MDD related to $G$ obtained from $\mathcal{H}$ by the corresponding transformation $\mathcal{T}_{1}$ or $\mathcal{T}_{2}$, then

- $l W_{a}=y W_{b} \Rightarrow l^{\prime} W_{a}>y^{\prime} W_{b}$,

- $h W_{b}=w W_{a} \Rightarrow h^{\prime} W_{b}>w^{\prime} W_{a}$.

Proof: Let us assume $h W_{b}=w W_{a}$. From Lemma 1-(f) it follows $l W_{a}>y W_{b}$. Then,

- If $l<2 w$, we have $\mathcal{H}^{\prime}=L(w, 2 h-y, 2 w-l, h)$ and so $h^{\prime} W_{b}>w^{\prime} W_{a} \Leftrightarrow l W_{a}>y W_{b}$.

- If $l>2 w>0$ with $l=\lfloor l / w\rfloor w+r$ and $0<r<w$, we have $\mathcal{H}^{\prime}=L(w,(\lfloor l / w\rfloor+1) h-$ $y, w-r, h)$. Therefore,

$h^{\prime} W_{b}>w^{\prime} W_{a} \Leftrightarrow\lfloor l / w\rfloor h W_{b}+r W_{a}>y W_{b} \Leftrightarrow(\lfloor l / w\rfloor w+r) W_{a}>y W_{b} \Leftrightarrow l W_{a}>y W_{b}$.

- If $l \geq 2 w>0$ with $l=\lfloor l / w\rfloor w$, we obtain $\mathcal{H}^{\prime}=L(w, l h / w-y, 0, h)$ and so

$$
h^{\prime} W_{b}>w^{\prime} W_{a} \Leftrightarrow(l h / w-y) W_{b}>0 \Leftrightarrow l W_{a}-y W_{b}>0 .
$$

If $l W_{a}=y W_{b}$, the proof is analogous to the previous case, now using Lemma 1-(e).

By Lemma 2 , transformations $\mathcal{T}_{1}$ and $\mathcal{T}_{2}$ can not be applied in any order. There are only two allowed compositions, $\mathcal{T}_{2} \circ \mathcal{T}_{1}$ or $\mathcal{T}_{1} \circ \mathcal{T}_{2}$, and both result in the identity transformation. 
Theorem 5 Let $\mathcal{H}=L(l, h, w, y)$ be an $M D D$ related to a digraph $G=G\left(N ; a, b ; W_{a}, W_{b}\right)$ with $\left(l W_{a}-y W_{b}\right)\left(h W_{b}-w W_{a}\right)=0$. Then, there are just two MDD related to $G$.

Proof: We first prove that if $\mathcal{H}^{\prime}=L\left(l^{\prime}, h^{\prime}, w^{\prime}, y^{\prime}\right)$ is the MDD related to $G$ obtained from $\mathcal{H}$ by the corresponding transformation $\mathcal{T}_{1}$ or $\mathcal{T}_{2}$, then any other MDD related to $G$ is contained in the region $\mathcal{H} \cup \mathcal{H}^{\prime}$. Let us assume $h W_{b}=w W_{a}$. Then, from Lemma 1-(f) we have $l W_{a}>y W_{b}$ and, by Lemma $2, h^{\prime} W_{b}>w^{\prime} W_{a}$. Hence, by Lemma $1-(\mathrm{a})$, the region $\nabla\left(0, h^{\prime}\right)=\nabla(0,2 h-y)$ can not contain any piece of some other MDD related to $G$. By items (b), (c) and (d) of Lemma 1, we also know that none of the regions $A_{\mathcal{H}}$ and $B_{\mathcal{H}}$ can intersect any other MDD related to $G$. Thus, any other MDD related to $G$ is contained in $\mathbb{N}^{2} \backslash\left(C_{\mathcal{H}^{\prime}} \cup A_{\mathcal{H}} \cup B_{\mathcal{H}}\right)=\mathcal{H} \cup \mathcal{H}^{\prime}$. The case $l W_{a}=y W_{b}$ can be proved by analogy.

Now we prove that there are just two MDD related to $G$. If $h W_{b}=w W_{a}$, then $\mathcal{H}^{\prime}=\mathcal{T}_{1}(\mathcal{H})$ follows from Theorem 3. Clearly $\mathcal{H}^{\prime}=\neq \mathcal{H}$. Analogously, if $l W_{a}=y W_{b}$ we have $\mathcal{T}_{2}(\mathcal{H}) \neq \mathcal{H}$. Therefore, we have related at least two MDD to $G$. We have to prove that there is no other MDD, $\mathcal{H}^{\prime \prime} \neq \mathcal{H}, \mathcal{H}^{\prime}$, related to $G$.

Let us assume $h W_{b}=w W_{a}\left(\mathcal{H}^{\prime}=\mathcal{T}_{1}(\mathcal{H})\right)$ and the subcase $l<2 w$ (see Figure 3$)$. Denote the rectangle $\{(m, n): i \leq m \leq s, j \leq n \leq t\}$ by $\rho[(i, j),(s, t)]$ whenever $i \leq s$ and $j \leq t$. Define the regions

$$
\begin{aligned}
\mathcal{E}_{1} & =\rho\left[\left(l^{\prime}, 0\right),(l-1, h-y-1)\right]=\rho[(w, 0),(l-1, h-y-1)], \\
\mathcal{E}_{2} & =\rho\left[(0, h),\left(l^{\prime}-w^{\prime}-1, h^{\prime}-1\right)\right]=\rho[(0, h),(l-w-1,2 h-y-1)], \\
\mathcal{F} & =\mathcal{H} \backslash \mathcal{E}_{1}=\Delta(w-1, h-y-1) \cup \Delta(l-w-1, h-1) .
\end{aligned}
$$

Regions $\mathcal{E}_{1}$ and $\mathcal{E}_{2}$ are the shadowed pieces appearing in the Figure 3 , and the region $\mathcal{F}$ is the lighter piece in the same figure.

Clearly $C_{\mathcal{H}}=\mathcal{E}_{2} \cup C_{\mathcal{H}^{\prime}}$ and $\mathcal{E}_{2}=\mathcal{E}_{1}+\boldsymbol{v}$. So, when applying $\mathcal{T}_{1}$ to $\mathcal{H}$ to obtain $\mathcal{H}^{\prime}$, region $\mathcal{F}$ remains fixed and region $\mathcal{E}_{1}$ is transformed into $\mathcal{E}_{2}$. Then, any other MDD $\mathcal{H}^{\prime \prime}$ related to $G$, different from $\mathcal{H}$ and $\mathcal{H}^{\prime}$, is contained in the region $\mathcal{R}=\mathcal{H} \cup \mathcal{H}^{\prime}=\mathcal{E}_{1} \cup \mathcal{F} \cup \mathcal{E}_{2}$.

Let us consider the unit squares $\llbracket w, 0 \rrbracket$ and $\llbracket 0, h \rrbracket=\llbracket w, 0 \rrbracket+\boldsymbol{v}$, which represent the same equivalence class $[w a]_{c}=[h b]_{c}$. The class $[w a]_{c}$ is only represented by these two unit squares in the region $\mathcal{R}$. Therefore, the MDD $\mathcal{H}^{\prime \prime}$ contains either $\llbracket w, 0 \rrbracket$ or $\llbracket 0, h \rrbracket$. If $\llbracket w, 0 \rrbracket \in \mathcal{H}^{\prime \prime}$, then $\nabla(0, h) \cap \mathcal{H}^{\prime \prime}=\emptyset$ because $\mathcal{H}^{\prime \prime}$ is an MDD. So, $\mathcal{H}^{\prime \prime}$ contains no unit square located in the region $\mathcal{E}_{2}$. Hence, $\mathcal{H}^{\prime \prime}=\mathcal{F} \cup \mathcal{E}_{1}=\mathcal{H}$. On the contrary, if $\llbracket 0, h \rrbracket \in \mathcal{H}^{\prime \prime}$, then $\mathcal{H}^{\prime \prime}=\mathcal{H}^{\prime}$. In any case, we conclude that the MDD $\mathcal{H}^{\prime \prime}$ is not different from either $\mathcal{H}$ or $\mathcal{H}^{\prime}$.

The other subcases, $l>2 w>0$ with $r>0$ and $l \geq 2 w>0$ with $r=0$, lead to the same conclussion with similar arguments.

If $l W_{a}=y W_{b}$ and $\mathcal{H}^{\prime}=\mathcal{T}_{2}(\mathcal{H})$, the statement can be proved by analogous reasonings.

\section{L-shapes associated to numerical 3-semigroups}

In this section, we first introduce some general classes of numerical semigroups useful in the study of curve singularities. Second, we associate L-shapes with numerical 3-semigroups and we describe how the main invariants of these semigroups are recognizable in their associated L-shapes. 


\subsection{Numerical semigroups}

A numerical semigroup $S$ is an additive subsemigroup of $\mathbb{N}$ with $0 \in S$. We can suppose that $\operatorname{gcd}(S)=1$, in other case we would take the quotient $S^{\prime}=S / \operatorname{gcd}(S)$. This condition is equivalent to the complementary of $S$ in $\mathbb{N}$ is finite, and we denote $\bar{S}=\mathbb{N}-S$ and $|\bar{S}|$, respectively the set and the number of gaps in the semigroup $S$. They are also equivalent to the existence of a conductor element $c(S)=\min \{x \in S: n \in S$ for any $n \geq x\}$ in $S$. The number $\mathfrak{f}(S)=c(S)-1$ is called the Frobenius number of $S$.

Numerical semigroups are finitely generated and any set of generators includes the minimal set of generators $S=\left\langle b_{0}, \ldots . ., b_{g}\right\rangle$, where $b_{0}=\min (S-\{0\})$ and $b_{i+1}=\min \left(S-\left\langle b_{0}, \ldots . ., b_{i}\right\rangle\right), i=$ $0, \ldots, g-1$. The Apéry set of $S$ respect the element $m \in S$ is $\operatorname{Ap}(S, m)=\{s \in S: s-m \notin S\}$. Then we have $\max \operatorname{Ap}(S, m)=\mathfrak{f}(S)-m$. For a up-to-date information about numerical semigroups see [12] and [9].

The classes of numerical semigroup that we will consider in what follows receives the name of its corresponding classes of associated curves.

In general, any numerical semigroup $S$ verifies that $m \in S \Longrightarrow c(S)-1-m \notin S$. A numerical semigroup $S$ is symmetric if satisfies $m \in S \Longleftrightarrow c(S)-1-m \notin S$. In this case the conductor verifies $c(S)=2|\bar{S}|[8]$.

A numerical semigroup $S=\left\langle b_{0}, \ldots . ., b_{g}\right\rangle$ is free if it verifies

$$
N_{i} b_{i} \in\left\langle b_{0}, \ldots, b_{i-1}\right\rangle, \text { para } i=1, \ldots, g
$$

where $N_{i}=e_{i-1} / e_{i}$ with $e_{i}=\operatorname{gcd}\left(b_{0}, \ldots, b_{i}\right), i=0,1, \ldots, g$.

A particular case of free semigroups are the so called plane curve semigroups arising as semigropus of values of analytically irreducible plane curve singularities. For the former, one has in addition

$$
N_{i} b_{i}<b_{i+1}, \quad i=1, \ldots, g-1
$$

In general, plane curve implies free and free implies symmetric.

Lemma 3 A numerical semigroup with two generators $S=\langle a, b\rangle$ is a plane curve semigroup, it have Apéry set $\operatorname{Ap}(S, a)=\{0, b, 2 b, \ldots,(a-1) b\}$ and conductor $c(S)=(a-1)(b-1)$.

A numerical semigroup with three generators $S=\langle a, b, c\rangle$ is a free semigroup if and only if $c \operatorname{gcd}(a, b) \in\langle a, b\rangle$ and it is a plane curve semigroup if furthermore $c\rangle \operatorname{lcm}(a, b)$.

Lemma 4 Let $S=\langle a, b, c\rangle$ be a minimally generated numerical semigroup with $1<a<b<$ $c$ and $\operatorname{gcd}(a, b)=p>1$. Considering $S_{p}=\langle a / p, b / p\rangle$ and $S^{\prime}=\langle a / p, b / p, c\rangle$ we have that:

(a) $S$ is symmetric if and only if $S^{\prime}$ is symmetric.

(b) $S$ is free if and only if $c \in S_{p}$.

(c) $S$ is plane curve if and only if $c \in S_{p}$ and $c>\operatorname{lcm}(a, b)$.

Proof: (a) is the Proposition 8 of [8], and (b) and (c) follow from Lemma 3. 


\subsection{L-shapes associated to numerical 3-semigroups}

The digraph $G^{*}=G(c ; a, b ; a, b)$ (i.e., the particular case $W_{a}=a$ and $\left.W_{b}=b\right)$ is closely related to the numerical 3-semigroup $S=\langle a, b, c\rangle$. Let $L$ be an MDD related to $G^{*}$ then, for each $\llbracket i, j \rrbracket \in L$, identity $M_{i a+j b}=i a+j b$ holds. In other words, the value $i a+j b$ is the minimum element of the equivalence class $[i a+j b]_{c}$ that belongs to the semigrup $S$. That is $i a+j b \in \operatorname{Ap}(c, S)$. This observation leads to the identity

$$
\left\{\mathrm{d}_{G^{*}}\left([0]_{c},[i a+j b]_{c}\right): \llbracket i, j \rrbracket \in L\right\}=\{i a+j b: \llbracket i, j \rrbracket \in L\}=\operatorname{Ap}(c, S) .
$$

Definition 2 An L-shape $L$ is associated to the numerical 3-semigroup $\langle a, b, c\rangle$ if $L$ is an $M D D$ related to the digraph $G(c ; a, b ; a, b)$.

Many properties of a numerical semigroup $S=\langle a, b, c\rangle$ can be derived from a related Apéry set. Note that the set of unit squares, by (3), gives the Apéry set $\operatorname{Ap}(c, S)$. Thus, some computations can be efficiently done from the related L-shape. See [2] for explicit obtention of sets of gaps. Computing the set of factorizations of $m \in S$, i.e. the set $\mathcal{F}(m, S)=\{(x, y, z) \in$ $\left.\mathbb{N}^{3}: a x+b y+c z=m\right\}$, via their related L-shapes can be found in [1]. The following result gives the Frobenius number and the number of gaps of a numerical 3 -semigroup from an associated L-shape.

Theorem 6 ([2]) Let $S=\langle a, b, c\rangle$ a numerical semigroup and $\mathcal{H}=L(l, h, w, y)$ an L-shape associated to $S$. Then

$$
\begin{gathered}
\mathfrak{f}(S)=\max \{(l-1) a+(h-y-1) b,(l-w-1) a+(h-1) b\}-c \\
2|\bar{S}|=\frac{l(h-y)}{c}[(l-1) a+(h-y-1) b]+\frac{y(l-w)}{c}[(l-w-1) a+(2 h-y-1) b]-c+1
\end{gathered}
$$

Note that the numbers $(l-1) a+(h-y-1) b$ and $(l-w-1) a+(h-1) b$ are the weights of the unit squares $\llbracket l-1, h-y-1 \rrbracket$ and $\llbracket l-w-1, h-1 \rrbracket$, i.e., the "dominant right interior corners" of the L-shape $\mathcal{H}$.

In the next section we will use the following result about the weight of the convex corner, i.e., the unit square $\llbracket l-w, h-y \rrbracket$, of an L-shape $L(l, h, w, y)$ associated to a semigroup.

Lemma 5 Let $\mathcal{H}=L(l, h, w, y)$ an L-shape associated to the numerical semigroup $S=$ $\langle a, b, c\rangle$, with $1<a<b<c$ and $\operatorname{gcd}(a, b, c)=1$. Then, if $h b=w a \quad(o r l a=y b)$ the weight of the convex corner of $\mathcal{H}$ is ac/h (or bc/h) and this weight does not change with the transformation $\mathcal{T}_{1}$ (or $\mathcal{T}_{2}$ ).

Proof: If $h b=w a$, the weight of the convex corner of $\mathcal{H}$ is $(l-w) a+(h-y) b=l a-y b=$ $a c / h$. Now we compute the weight of the convex corner of $\mathcal{T}_{1}(\mathcal{H})=\mathcal{H}^{\prime}$ by cases.

- If $l<2 w$, the convex corner preserves its position and its weight, since $\mathcal{H}^{\prime}=L(w, 2 h-$ $y, 2 w-l, l)$ and its convex corner is $\llbracket w-(2 w-l), 2 h-y-l \rrbracket=\llbracket l-w, h-y) \rrbracket$.

- If $l \geq 2 w$ and $w$ divides to $l$, we have $\mathcal{H}^{\prime}=L(w, l h / w-y, 0, h)$ and its convex corner is $\llbracket w, l h / w-y-h \rrbracket$ with weight $w a+(l h / w-y-h) b=l a-y b$.

- If $l \geq 2 w$ and $l=\lfloor l / w\rfloor w+r$ with $0<r<w$, we have $\mathcal{H}^{\prime}=L(w,(\lfloor l / w\rfloor+1) h-y, w-r, h)$ and its convex corner is $\llbracket w-(w-r),(\lfloor l / w\rfloor+1) h-y-h \rrbracket=\llbracket r,\lfloor l / w\rfloor h-y \rrbracket$ with weight $r a+(\lfloor l / w\rfloor h-y) b=r a+\lfloor l / w\rfloor w a-y b=l a-y b$. 
Lemma 6 Let $\mathcal{H}=L(l, h, w, y)$ an L-shape associated to the numerical semigroup $S=$ $\langle a, b, c\rangle$, with $1<a<b<c$ and $\operatorname{gcd}(a, b, c)=1$. Consider $m, n \in \mathbb{N}$ with $\operatorname{gcd}(m, n)=$ $\operatorname{gcd}(m, c)=1, n|a, n| b$ and $m b<n c$. Then $\mathcal{H}$ is also related to the 3 -numerical semigroup $S^{\prime}=\left\langle a \frac{m}{n}, b \frac{m}{n}, c\right\rangle$.

Proof: Obviously, condition (1) and Theorem 1, taking $W_{a}=a$ and $W_{b}=b$, are fulfilled by $\mathcal{H}$ and $S^{\prime}$.

Consider the congruence $l a \equiv y b(\bmod c)$ in $(2)$, then there is some $\lambda \in \mathbb{Z}$ with $l a-y b=\lambda c$. From $n \mid(l a-y b)$ and $\operatorname{gcd}(n, c)=1$, we have $n \mid \lambda$ and then $m\left(l \frac{a}{n}-y \frac{b}{n}\right)=m \frac{\lambda}{n} c$ and so $l a \frac{m}{n} \equiv y b \frac{m}{n}$ $(\bmod c)$ holds. The second congruence $h b \frac{m}{n} \equiv w a \frac{m}{n}(\bmod c)$ follows from the same argument. Thus, condition (2) holds for $\mathcal{H}$ and $S^{\prime}$.

\section{Classification of numerical 3-semigroups by means of L-shapes}

In this section, we characterize the numerical 3-semigroups considered in the previous section, in terms of the parameters of their associated L-shapes.

Next theorem gives a first characterization of symmetric 3-semigroups by means of the parameters of an associated L-shape.

Theorem 7 Let $S=\langle a, b, c\rangle$ a numerical semigroup and $\mathcal{H}=L(l, h, w, y)$ an L-shape associated to $S$. Then, $S$ is symmetric if and only if $w y(l a-y b)(w a-h b)=0$.

Proof: If $(w, y)=(0,0)$, from (4) and (5), we have $2|\bar{S}|=\mathfrak{f}(S)+1$ and so $S$ is symmetric. In other case, we can write the expression (4) as

$$
\mathfrak{f}(S)=(l-w-1) a+(h-y-1) b+\max \{w a, y b\}-c
$$

Using (5), (6) and $c=l h-w y$ we have

- If $w a=y b$, then $\llbracket w, 0 \rrbracket$ and $\llbracket 0, y \rrbracket$ belong to $\mathcal{H}$ and have the same weight, so $w y=0$.

- If $w a>y b$, then

$$
\begin{aligned}
S \text { symmetric } & \Leftrightarrow 2|\bar{S}|=\mathfrak{f}(S)+1 \\
& \Leftrightarrow(l-w) y(h b-w a)=0 \\
& \Leftrightarrow y(h b-w a)=0, \text { since } 0<w<l .
\end{aligned}
$$

- If $w a<y b$, in the same way, $S$ symmetric if and only if $w(l a-y b)=0$.

So, if $S$ is symmetric then $w y(l a-y b)(w a-h b)=0$. Reciprocally, note that $w=0$ implies $w a<y b$ and also $l a-y b=0$ implies $w a<y b$. Analogously, $y(w a-h b)=0$ implies $w a>y b$. Then, in any case, the statement follows from items (e) and (f) of Lemma 1.

As a consequence of this result, a symmetric 3-semigroup has associated an L-shape $\mathcal{H}=$ $L(l, h, w, y)$ with $w y=0$, in which case $\mathcal{H}$ is rectangular but not unique, or $(l a-y b)(w a-$ $h b)=0$, in which case $S$ has associated two L-shapes, where only one of them can be rectangular (Theorems 3 and 4 ). 
Note that in the characterization of symmetric 3-semigroups given in Theorem 7 it is not required that $\{a, b, c\}$ be a minimal system of generators for $S$. In this section, we classify numerical semigroups introduced in Section 4 in the case 3-generated, $S=\langle a, b, c\rangle$, and we do it in function on the number and the degeneration or not of their associated L-shapes. This classification basically depends on whether $\{a, b, c\}$ is a minimal system of generators for $S$ or not, and on whether the terms $a, b$ and $c$ are coprime or not.

First of all, we consider the case $1<a<b<c$ with $\operatorname{gcd}(a, b, c)=1$ and $\{a, b, c\}$ is not a minimal system of generators of $S$. In these conditions, $\operatorname{gcd}(a, b) \in\{1, a\}$.

If $\operatorname{gcd}(a, b)=1$, then $c=\lambda a+\mu b, \lambda, \mu \in \mathbb{N}$ and we have for $S$ the following result.

Theorem 8 Let $S=\langle a, b, c\rangle$ a numerical semigroup with $1<a<b<c, \operatorname{gcd}(a, b)=1$ and $c=\lambda a+\mu b$ with $\lambda, \mu \in \mathbb{N} \cup\{0\}$. We have:

(a.1) If $c=\lambda a$ with $\lambda \leq b$, then $S$ has associated a unique rectangular $L$-shape $\mathcal{H}=$ $L(\lambda, a, 0,0)$ if $\lambda$ divides to $b$, and $\mathcal{H}=L(\lambda, a, \lambda-r, 0)$, with $\lambda-r \equiv b(\bmod \lambda)$, if not.

(a.2) If $c=\lambda a$ with $\lambda>b$, then $S$ has associated a rectangular $L$-shape $\mathcal{H}=L(\lambda, a, b, 0)$ and other $\mathcal{H}^{\prime}=\mathcal{T}_{1}(\mathcal{H})$ that can be or not rectangular (also $\mathcal{H}=\mathcal{T}_{2}\left(\mathcal{H}^{\prime}\right)$ ).

(b.1) If $c=\mu b$ with $\mu \leq a$, then $S$ has associated a unique rectangular $L$-shape $\mathcal{H}=$ $L(b, \mu, 0,0)$ if $\mu$ divides to $a$, and $\mathcal{H}=L(b, \mu, 0, \mu-r)$, with $\mu-r \equiv a(\bmod \mu)$, if not.

(b.2) If $c=\mu b$ with $\mu>a$, then $S$ has associated a rectangular $L$-shape $\mathcal{H}=L(b, \mu, 0, a)$ and other $\mathcal{H}^{\prime}=\mathcal{T}_{2}(\mathcal{H})$ that can be or not rectangular (also $\mathcal{H}=\mathcal{T}_{1}\left(\mathcal{H}^{\prime}\right)$ ).

(c) $S$ has associated two rectangular L-shapes if and only if $c=\lambda a=\mu b>a b$.

(d) If $c=\lambda a+\mu b$ such that $a$ and $b$ does not divide to $c$, then $S$ has associated two non rectangular $L$-shapes $\mathcal{H}=L\left(\lambda^{\prime}+b, a, b, a-\mu^{\prime}\right)$, being $c=\lambda^{\prime} a+\mu^{\prime} b$ with $1 \leq \mu^{\prime}<a$, and $\mathcal{H}^{\prime}=\mathcal{T}_{1}(\mathcal{H})$ (also $\mathcal{H}=\mathcal{T}_{2}\left(\mathcal{H}^{\prime}\right)$ ).

Proof: In each case, we prove that the corresponding L-shapes verify the compatibility conditions (1) and (2), and Theorem 1.

(a.1) There exists $r, 0 \leq r<\lambda$, such that $b+r \equiv 0(\bmod \lambda)$ and so $a b+a r \equiv 0(\bmod \lambda a)$ (see left hand side of Figure 6). Note that $r=0$ if and only if $\lambda$ divides to $b$.

For (1), if $\operatorname{gcd}(\lambda, a, \lambda-r)=g>1$, then $\operatorname{gcd}(\lambda, a, r)=g$ and $g$ divides to $b$, thus we obtain the contradiction $\operatorname{gcd}(a, b) \geq g>1$.

For Theorem 1, we have $l a-y b=\lambda a>0$ and $h b-w a=a(b-\lambda+r)>0$, since $0<$ $r<\lambda$, which also proves the unicity of this L-shape. To verfy the rest conditions is a simple inspection.

(a.2) If $\lambda>b$, the situation is represented in the right hand side of Figure 6, where the zero $[\lambda a]_{\lambda a}$ in the unit square $\llbracket \lambda, 0 \rrbracket$ is repeated in the unit square $\llbracket \lambda-b, a \rrbracket$. Hence, an associated L-shape is $\mathcal{H}=L(\lambda, a, b, 0)$.

Conditions (1) and (2) are clear. Now we have $l a-y b=\lambda a>0$ and $h b-w a=a b-b a=0$. Then Theorem 1 and Theorem 5 assure the statement.

In a similar way we can prove (b.1) and (b.2). 

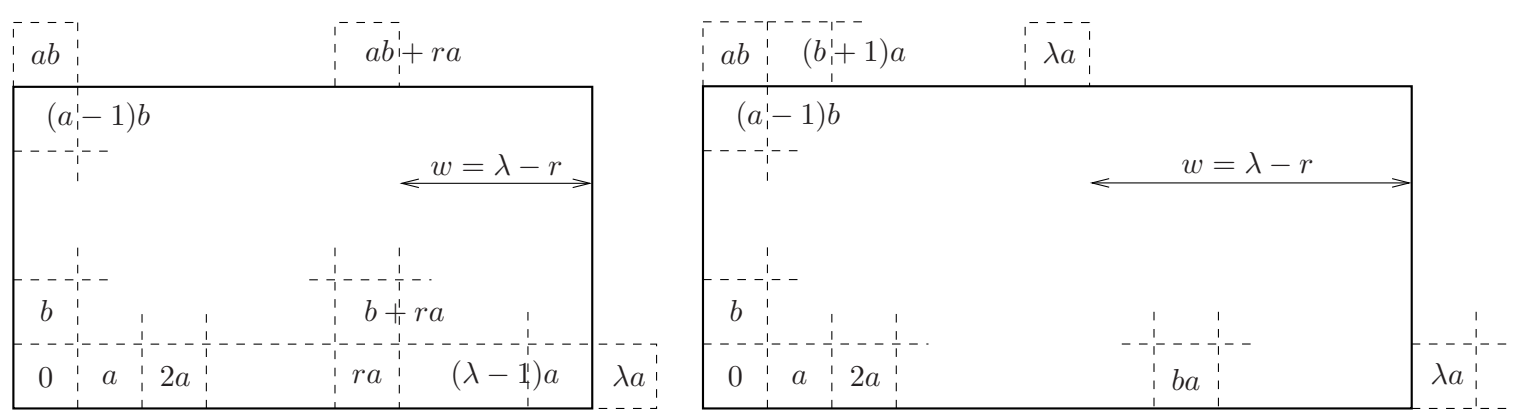

Figure 6: Diagrams of $L(\lambda, a, \lambda-r, 0)$ and $L(\lambda, a, b, 0)$

(d) We have $c=\lambda a+\mu b=(\lambda+k b) a+(\mu-k a) b$ for any $k \in \mathbb{Z}$. Since $a$ does not divide to $c$, there exists $k \in \mathbb{N} \cup\{0\}$ such that $0<\mu-k a<a$. Denoting $\mu^{\prime}=\mu-k a$ and $\lambda^{\prime}=\lambda+k b$, we can write $c=\lambda^{\prime} a+\mu^{\prime} b$ with $1 \leq \mu^{\prime}<a$.

Let see that $\mathcal{H}=L\left(\lambda^{\prime}+b, a, b, a-\mu^{\prime}\right)$ is a non rectangular $\left(\mu^{\prime}<a\right)$ L-shape associated to $S$, how is showed in Figure 7. Conditions (1) are clear. From $l a-y b=\left(\lambda^{\prime}+b\right) a-\left(a-\mu^{\prime}\right) b=$ $\lambda^{\prime} a+\mu^{\prime} b \equiv 0(\bmod c)$ and $h b-w a=a b-b a=0 \equiv 0(\bmod c)$ follow conditions $(2)$. Since $l a-y b>0$, the L-shape $\mathcal{H}$ verifies Theorem 1 , and Theorem 3 gives the other L-shape $\mathcal{H}^{\prime}=L\left(b, \mu^{\prime \prime}+a, b-\lambda^{\prime \prime}, a\right)$, where $c=\lambda^{\prime \prime} a+\mu^{\prime \prime} b$ with $1 \leq \lambda^{\prime \prime}<b$.

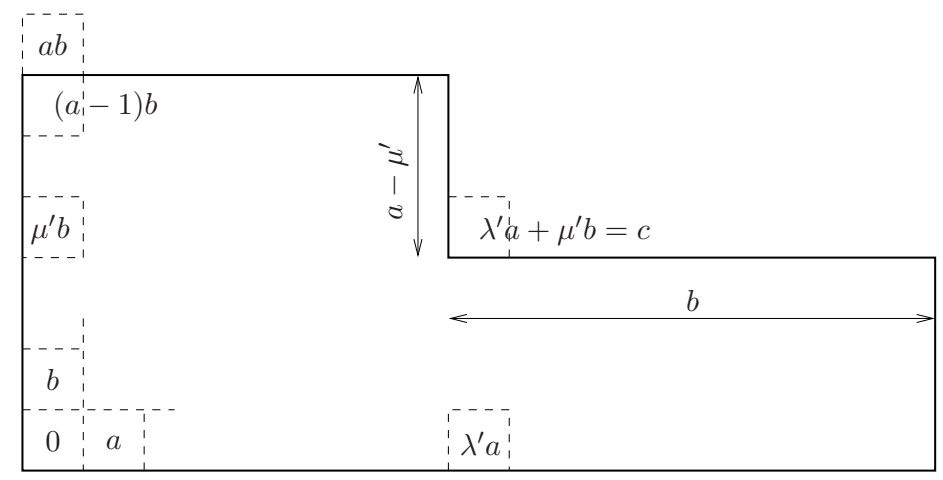

Figure 7: Diagram of $\mathcal{H}=L\left(\lambda^{\prime}+b, a, b, a-\mu^{\prime}\right)$

(c) As $c=\lambda a=\mu b>a b$, by exclusion, we are simultanesly in the cases (a.2) and (b.2). So $S$ has associated the L-shapes $\mathcal{H}=L(\lambda, a, b, 0)$ and $\mathcal{H}^{\prime}=L(b, \mu, 0, a)$. Obviously $\mathcal{T}_{1}(\mathcal{H})=\mathcal{H}^{\prime}$ and $\mathcal{T}_{2}\left(\mathcal{H}^{\prime}\right)=\mathcal{H}$.

If $\operatorname{gcd}(a, b)=a$, then $b=k a, k \in \mathbb{N}$ and $\operatorname{gcd}(a, c)=1$. In this case, we have for $S$ the following result.

Theorem 9 Let $S=\langle a, b, c\rangle$ a numerical semigroup with $1<a<b<c, \operatorname{gcd}(a, b, c)=1$ and $b=k a$. Then:

(a) $S$ has associated two L-shapes and at least one of them is the rectangular $L$-shape $\mathcal{H}=L(c, 1, k, 0)$.

(b) $S$ has associated two degenerated L-shapes if and only if $k$ divides to $c$. The other rectangular $L$-shape associated to $S$ is $\mathcal{T}_{1}(\mathcal{H})=L(k, c / k, 0,1)$. 
(c) If $k$ does not divide to $c$, the other non rectangular $L$-shape associated to $S$ is $L(k,\lfloor c / k\rfloor+$ $1, k-r, 1)$, with $c=\lfloor c / k\rfloor k+r$ and $0<r<k$.

Proof: $\operatorname{As} \operatorname{gcd}(a, c)=1$, the zero $[c a]_{c}$ is in the unit squares $\llbracket c, 0 \rrbracket$ and $\llbracket c-k, 1 \rrbracket$. So $\mathcal{H}=L(c, 1, k, 0)$, verifying conditions (1) and (2), and Theorem 1 , is an associated L-shape to $S$. Furthermore, $h b-w a=0$ and Theorem 3 gives the other L-shape $\mathcal{H}^{\prime}=\mathcal{T}_{1}(\mathcal{H})$, which completes part (a).

If $k$ divides to $c$, then $\mathcal{T}_{1}(\mathcal{H})=L(k, c / k, 0,1)$. As $c>2 k$ in $\mathcal{H}$, the other unique possible $\mathcal{T}_{1}(\mathcal{H})$ corresponds to the case in which $k$ does not divide to $c$. If $c=\lfloor c / k\rfloor k+r$, with $0<r<k$, then $\mathcal{T}_{1}(\mathcal{H})=L(k,\lfloor c / k\rfloor+1, k-r, 1)$. This new L-shape is non rectangular since $0<r<k$. This prove (b) and (c).

Remark 1 The geometric classification of numerical 3-semigroups whose system of generators is not minimal is irrelevant since, in fact, they are semigroups 2-generated and, in particular, they are plane curve semigroups (Lemma 3). Theorems 8 and 9 can be used as a classification of numerical 2-semigroups in terms of their associated L-shapes, and we next use them to classify numerical 3-semigroups.

Now we consider numerical 3-semigroups $S$ given by a minimal system of generators $\langle a, b, c\rangle$, and we discern the cases $\operatorname{gcd}(a, b)=1$ and $\operatorname{gcd}(a, b)>1$.

Remark 2 If $S=\langle a, b, c\rangle$ is a numerical semigroup with $1<a<b<c, \operatorname{gcd}(a, b)=1$ and $c \notin\langle a, b\rangle$, then $c$ only can take values in the set of gaps of the semigroup $S^{\prime}=\langle a, b\rangle$. In particular $b<c<(a-1)(b-1)$. Therefore there are a finite number of numerical semigroups $S$ under the described conditions. The following theorem characterizes them in terms of their asociated L-shapes.

Theorem 10 Let $S=\langle a, b, c\rangle$ a numerical semigroup with $1<a<b<c, \operatorname{gcd}(a, b)=1$ and $c \notin\langle a, b\rangle$. Then,

(a) $S$ is non symmetric if and only if $S$ has associated a unique non rectangular $L$-shape.

(b) $S$ is symmetric if and only if $S$ has associated a unique rectangular L-shape $\mathcal{H}$ where:

(b.1) if $\operatorname{gcd}(a, c)=p>1$ and $\operatorname{gcd}(b, c)=1$, then $\mathcal{H}=L\left(\frac{c}{p}, p, 0,0\right)$ or $\mathcal{H}=L\left(\frac{c}{p}, p, \frac{c}{p}-\right.$ $k, 0)$ with $0<k<\frac{c}{p}$ and $b+k \frac{a}{p} \equiv 0\left(\bmod \frac{c}{p}\right)$;

(b.2) if $\operatorname{gcd}(a, c)=1$ and $\operatorname{gcd}(b, c)=q>1$, then $\mathcal{H}=L\left(q, \frac{c}{q}, 0,0\right)$ or $\mathcal{H}=L\left(q, \frac{c}{q}, 0, \frac{c}{q}-\right.$ k) with $0<k<\frac{c}{q}$ and $a+k \frac{b}{q} \equiv 0\left(\bmod \frac{c}{q}\right)$;

(b.3) if $\operatorname{gcd}(a, c)=p>1$ and $\operatorname{gcd}(b, c)=q>1$, then $\mathcal{H}$ is as in (b.1) if $a q<b p$ or as in (b.2) if $a q>b p$.

(c) $S$ is non free.

\section{Proof:}

(a) Follows from Theorem 7.

(b) It is well known [8] that if $S=\langle a, b, c\rangle$ is a symmetric semigroup, then the generators $a, b$ and $c$ can not have any coprime pairs, thus $\operatorname{gcd}(a, c)>1$ or $\operatorname{gcd}(b, c)>1$. 
If $\operatorname{gcd}(a, c)=p>1$, then $a / p$ and $c / p$ are coprime and, so, there exists $k$ with

$$
0 \leq k \leq \frac{c}{p} \text { such that } b+k \frac{a}{p} \equiv 0\left(\bmod \frac{c}{p}\right)
$$

and so $p b+k a \equiv 0(\bmod c)$. This minds that we have a zero $[0]_{c}$ in the unit square $\llbracket k, p \rrbracket$. On the other hand, since $\operatorname{lcm}(a, c)=a c / p$ there is another zero $[0]_{c}$ in the unit square $\llbracket c / p, 0 \rrbracket$.

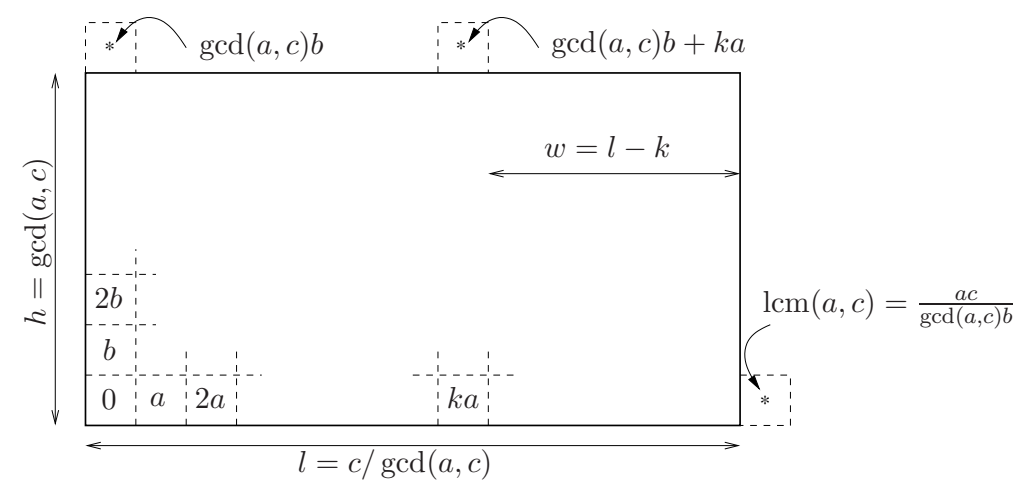

Figure 8: $L(c / \operatorname{gcd}(a, c), \operatorname{gcd}(a, c), c / \operatorname{gcd}(a, c)-k, 0)$

The rectangle $L(l, h, w, 0)$ of the Figure 8 with $l=c / p, h=p \quad \mathrm{y} \quad w=l-k$ is an L-shape associated to $S$. This L-shape is unique because $l a-y b=l a>0$ and $h b-w a=p b+k a-\operatorname{lcm}(a, c)>0$ since $p b+k a$ is the zero $[0]_{c}$ in the unit square $\llbracket k, p \rrbracket$ and $\operatorname{lcm}(a, c)$ is the zero $[0]_{c}$ in the unit square $\llbracket c / p, 0 \rrbracket$, and both have distinct weight.

If $\operatorname{gcd}(b, c)=q>1$ we obtain analogously the unique L-shape $L(l, h, 0, y)$ with $l=$ $q, h=c / q, y=h-k$ and

$$
0 \leq k \leq \frac{c}{q} \text { such that } a+k \frac{b}{q} \equiv 0\left(\bmod \frac{c}{q}\right)
$$

In the hypothesis (b.3) we have $\operatorname{gcd}(p, q)=1$ and $a q \neq b p$, since if $a q=b p$ then $\operatorname{gcd}(a, b)=a / p=b / q=1$ implies $a=p$ and $b=q$. In which case we have $c \geq p q$, a contradiction with $b<c<(a-1)(b-1)$ (Remark 2).

(c) Follows from Lemma 3 and the hypothesis $c \notin\langle a, b\rangle$.

Remark 3 Can occur that $\operatorname{gcd}(a, c)>1$ and $\operatorname{gcd}(b, c)>1$, in which case we have two $L$ shapes. Then, either one of them is not a DDM, as it happens with the semigroup $\langle 4,15,18\rangle$ having an $L$-shape $L(9,2,3,0)$ that is a DDM and another $L$-shape $L(3,6,0,2)$ that is not, either both L-hapes coincide. This occur if $k=0$, in which case $l-k \equiv 0(\bmod l)$. For example, the symmetric semigroup $\langle 8,15,20\rangle$ has the same $L$-shape $L(5,4,0,0)$ in both cases.

Example 1 Let $S_{c}=\langle 6,7, c\rangle$ with $\operatorname{gcd}(6,7, c)=1$ and $\left.c\right\rangle 7$. Then, the term $c$ takes values in the set $L \cup T$ where $L=\{8,9,10,11,15,16,17,22,23,29\}$ is the set of gaps of the semigroup $\langle 6,7\rangle$ (that are) greater than 7 , and $T$ is the set of terms of the semigroup $\langle 6,7\rangle$ greater than 7 .

- If $c \in T$, then $S_{c}$ is ruled by Theorem 8: 
(a.1) If $c=6 \lambda$ with $\lambda \leq 7$, then $\mathcal{H}_{c}=L(\lambda, 6, \lambda-r, 0)$ with $\lambda-r \equiv 7(\bmod \lambda), 0 \leq r<\lambda$.

(a.2) If $c=6 \lambda$ with $\lambda>7$ and $\lambda=7 p+r, p \geq 1,0<r<7$, then $\mathcal{H}_{c}=L(\lambda, 6,7,0)$ and $\mathcal{T}_{1}\left(\mathcal{H}_{c}\right)=L(7,6(p+1), 7-r, 6)$.

(b.1) If $c=7 \mu$ with $\mu \leq 6$, then $\mathcal{H}_{c}=L(7, \mu, 0,7-r)$ with $\mu-r \equiv 6(\bmod \mu), 0 \leq r<\mu$.

(b.2) If $c=7 \mu$ with $\lambda>6$ and $\mu=6 p+r, p \geq 1,0<r<6$, then $\mathcal{H}_{c}=L(7, \mu, 0,6)$ and $\mathcal{T}_{2}\left(\mathcal{H}_{c}\right)=L(7(p+1), 6,7,6-r)$.

(c) If $c=42 k$ with $k>1$, then $\mathcal{H}_{c}=L(7 k, 6,7,0)$ and $\mathcal{H}_{c}^{\prime}=L(7,6 k, 0,6)$.

(d) If $c=6 \lambda+7 \mu$ with $1 \leq \mu<6$ and $\lambda=7 k+r$ with $0<r<7$, then $\mathcal{H}_{c}=$ $L(\lambda+7,6,7,6-\mu)$ and $\mathcal{T}_{1}\left(\mathcal{H}_{c}\right)=L(7,6(k+1)+\mu, 7-r, 6)$.

- If $c \in L$ then $S_{c}$ is ruled by Theorem 10: the semigroups $\langle 6,7,8\rangle,\langle 6,7,9\rangle$ and $\langle 6,7,15\rangle$ have, respectively, a unique rectangular $L$-shape $L(4,2,1,0), L(3,3,2,0)$ and $L(5,3,1,0)$ and, so, they are symmetric.

For the values $c=10,11,16,17,22,23$ and 29 we have, respectively, unique non rectangular $L$-shapes $L(4,4,3,2), L(3,4,1,1), L(5,4,2,2), L(4,5,3,1), L(6,4,1,2), L(5,5,2,1)$ and $L(6,5,1,1)$ and, so, they are non symmetric.

Example 2 Each numerical semigroup $S_{c}=\langle 4,12, c\rangle$, with $\operatorname{gcd}(4,12, c)=1$ and $c>12$, has associated a rectangular $L$-shape $\mathcal{H}_{c}=L(c, 1,3,0)$. The other $L$-shape depends on that 3 divides or not to $c$.

(b) If 3 divides to $c$, then $c=3 q$ with $q \geq 5$ odd, i.e., $c=15,21,27, \ldots$, and the other $L$-shape is $\mathcal{T}_{1}\left(\mathcal{H}_{c}\right)=L(3, q, 0,1)$.

(c) If 3 does not divide to $c$ we discern the cases:

$c=3 q+1$ with $q \geq 6$ even, i.e., $c=19,25,31, \ldots$, and the other $L$-shape is $\mathcal{T}_{1}\left(\mathcal{H}_{c}\right)=$ $L(3, q+1,2,1)$.

$c=3 q+2$ with $q \geq 5$ odd, i.e., $c=17,23,29, \ldots$, and the other $L$-shape is $\mathcal{T}_{1}\left(\mathcal{H}_{c}\right)=$ $L(3, q+1,1,1)$.

Theorem 11 Let $S=\langle a, b, c\rangle$ be a minimally generated numerical semigroup with $1<a<$ $b<c$ and $\operatorname{gcd}(a, b)=p>1$. Considering $S_{p}=\langle a / p, b / p\rangle$ and $S^{\prime}=\langle a / p, b / p, c\rangle$ we have that:

(a) $S$ is non symmetric if and and if $S^{\prime}$ has associated a unique non rectangular $L$-shape.

(b) $S$ is symmetric but no free if and and if $S^{\prime}$ has associated a unique rectangular L-shape as it has been described in Theorem 10 (b).

(c) $S$ is free if and and if the associated L-shapes to $S^{\prime}$ are ruled by Theorem 8.

(d) $S$ is plane curve if and and if $S$ is free and the weight of the convex corner $(l-w, h-y)$ of any associated $L$-shape to $S^{\prime}$ is greater than $\operatorname{lcm}(a, b)$.

Furthermore, in all cases, the semigroups $S$ and $S^{\prime}$ have associated the same L-shapes. In each case, these L-shapes are given by the corresponding theorems 10 and 8. 
Proof: Clearly $1<a / p<b / p<c$ and $\operatorname{gcd}(a / p, b / p)=1$. If $S$ is not free, then $c \notin S_{p}$ and, so, $S^{\prime}$ verifies the hypothesis of the Theorem 10 . It proves the parts (a) and (b).

On the contrary, if $S$ is free, then $c \in S_{p}$ by Lemma 4 , and hence $S^{\prime}$ is described by Theorem 8 . Finally, note that the weight of the convex corners of the associated L-shapes to the semigroups $S$ and $S^{\prime}$ are $c p$ and $c$, respectively, and from Lemma 4 we conclude $c>\operatorname{lcm}(a, b)$.

That $S$ and $S^{\prime}$ have the same L-shapes follows from Lemma 6.

Note that, from Theorems 10 and 11, all minimally generated numerical 3-semigroups, excepting a finite number, are free.

Example 3 Let $S_{c}=\langle 12,14, c\rangle$, with $c>14$ and $\operatorname{gcd}(12,14, c)=1$, and let $S_{c}^{\prime}=\langle 6,7, c\rangle$. The semigroup $S_{c}$ is ruled by Theorem 11:

(a) $S_{c}$ is non symmetric if and only if $c=17,23,29$, with respective $L$-shapes $L(4,5,3,1)$, $L(5,5,2,1), L(6,5,2,1)$.

(b) $S_{c}$ is symmetric but non free if and only if $c=15$, with $L$-shape $L(5,3,1,0)$.

(c) Following Theorem 8 and Example 1, $S_{c}$ is free:

(b1) if $c=21,35$, with respective $L$-shapes $L(7,3,0,7), L(7,5,0,1)$.

(b2) if $c=7 \mu$ with $\mu \geq 7$ odd and $\mu=6 p+r$ with $p \geq 1$ and $0<r<6$, with two $L$-shapes $\mathcal{H}_{c}=L(7, \mu, 0,6)$ and $\mathcal{T}_{2}\left(\mathcal{H}_{c}\right)=L(7(p+1), 6,7,6-r)$.

(d) if $c=6 \lambda+7 \mu$ with $\mu=1,3,5$ and $\lambda=7 k+r$ with $0<r<7$, with two $L$-shapes $\mathcal{H}_{c}=L(\lambda+7,6,7,6-\mu)$ and $\mathcal{T}_{1}\left(\mathcal{H}_{c}\right)=L(7,6(k+1)+\mu, 7-r, 6)$.

(d) The free semigroups of (c) are plane curve if and only if $c \geq 85$.

\section{References}

[1] F. Aguiló-Gost and P.A. García Sánchez, Factoring in Embedding Dimension Three Numerical Semigroups, Electronic J. of Combin. 17 (2010) \#R138.

[2] F. Aguiló, A. Miralles and M. Zaragozá, Using Double-Loop digraphs for solving Frobenius' Problems, ENDM 24 (2006) 17-24.

[3] F. Aguiló and A. Miralles, Familias infinitas de digrafos de Cayley de grado 2 óptimos sobre grupos abelianos finitos, Actas JMDA2012 5 (2012) 29-36.

[4] A. Campillo and C. Marijuán, Higher order relations for a numerical semigroup, Séminaire de Théorie des Nombres, Bordeaux 3 (1991), 249-260.

[5] C. Delorme, Sous-monoïdes d'intersection compléte de $\mathbb{N}$, Ann. Scient. Ec. Norm. Sup. 9 (1976) 145-154.

[6] P. Esqué, F. Aguiló and M.A. Fiol, Double commutative-step digraphs with minimum diameters, Discrete Math. 114 (1993) 147-157.

[7] M.A. Fiol, J.L.A. Yebra, I. Alegre and M. Valero, A discrete optimization problem in local networks and data alignment, IEEE Trans. Comput. C-36 (1987) 702-713. 
[8] R. Fröberg, C. Gotlieb and R. Häggkvist, On numerical semigroups Semigroup Forum 35 (1987) 63-83.

[9] J.L. Ramírez Alfonsín, The Diophantine Frobenius Problem, Oxford Univ. Press, Oxford, ISBN 978-0-19-856820-9 (2009).

[10] Ø.J. Rødseth, On a linear diophantine problem of Frobenius, J. Reine Angewandte Math. 301 (1978) 171-178.

[11] Ø.J. Rødseth, Weighted multi-connected loop networks, Discrete Mathematics 148 (1996) 161-173.

[12] J.C. Rosales and P.A. García-Sánchez, Numerical semigroups, Developments in Mathematics, 20. Springer, New York, ISBN: 978-1-4419-0159-0 (2009). 